\title{
Measuring convergence of mixed finite element discretizations: an application to shell structures
}

\author{
Jean-François Hiller, Klaus-Jürgen Bathe * \\ Department of Mechanical Engineering, Massachusetts Institute of Technology, 77 Massachusetts Avenue, \\ Room 3-356, Cambridge, MA 02139, USA
}

\begin{abstract}
We consider the problem of assessing the convergence of mixed-formulated finite elements. When displacementbased formulations are considered, convergence measures of finite element solutions to the exact solution of the mathematical problem are well known. However when mixed formulations are considered, there is no well-established method to measure the convergence of the finite element solution. We first review a number of approaches that have been employed and discuss their limitations. After having stated the properties that an ideal error measure would possess, we introduce a new physics-based procedure. The new proposed error measure can be used for many different types of mixed formulations and physical problems. We illustrate its use in an assessment of the performance of the MITC family of shell elements.
\end{abstract}

(C) 2003 Elsevier Science Ltd. All rights reserved.

\section{Introduction}

For many analyses of engineering interest, displacement-based finite element formulations are most effective. These formulations have some important advantages and most notably a strong mathematical background and simplicity of implementation. For various types of problems, however, analysts have to resort to mixed formulations $[1,2]$ because no reliable and efficient displacement-based formulation exists. The analysis of incompressible and near-incompressible solids is a typical example. Whereas the low-order classical displacementbased finite elements perform very well when compressible materials are considered, the phenomenon of locking appears when the same elements are used to model incompressible and near-incompressible materials. This phenomenon manifests itself in the form of an excessively stiff behavior of the finite element model when the Poisson ratio approaches the value 0.5 . More formally, we say that a finite element formulation dis-

\footnotetext{
${ }^{*}$ Corresponding author. Tel.: +1-617-253-6645; fax: +1-617253-2275.

E-mail address: kjb@mit.edu (K.J. Bathe).
}

plays locking if the convergence of the finite element solution to the exact solution of the underlying mathematical model - in the appropriate norm - is not uniform and optimal. Locking results from the relative inability of the finite element space to approximate the exact solution which is constrained - in this case by the incompressibility condition $\operatorname{div}(U)=0$.

Similar locking phenomena are observed when displacement(velocity)-based finite element techniques are employed to solve for the displacements of thin plates or shells, in contact problems, in incompressible fluids, and so on. In each case, the constraint on the exact solution is different: vanishing shear strains in plates, vanishing membrane and transverse shear strains in bendingdominated situations for shells, the no-penetration constraint in contact problems, the incompressibility condition in fluids. In all cases, the effective analysis of these problems requires the use of an alternative approach to displacement-based finite element formulations. Mixed-interpolated finite elements have been proposed as that alternative and are widely used both in academic research and industry [1-3].

However, for each of the mentioned types of analysis there often exist several competing mixed formulations and new ones are still being developed. Under these 
circumstances, the need for an objective means to test the performance of mixed finite element methods is apparent.

If mixed formulations tend to behave better than displacement-based ones in some cases, the mathematics underlying mixed formulations is significantly more complex. As a result, although the mathematical conditions (in particular the inf-sup condition [3]) that a mixed scheme need to satisfy for uniform optimality in the appropriate norm are well known, there is frequently no available analytical proof whether these conditions are satisfied by mixed-formulated elements if realistic problems are considered.

In the absence of an analytical proof of optimality, numerical tests need to be carried out to validate existing and new mixed finite element formulations. Some such studies have focused on the convergence of pointwise values of finite element fields to assess the effectiveness of finite elements. Although such studies are rather straightforward to carry out and can be employed to test for the simpler forms of locking, they do little to help determine whether or not a formulation locks in the stricter sense, i.e. whether the convergence rate is optimal and uniform.

In shell analysis, there is the added difficulty of having to select appropriate test problems that reflect the entire range of possible load-carrying mechanisms, asymptotic behaviors and surface geometries [4-9]. If inappropriate or insufficient test problems are used, incorrect conclusions about the performance of a discretization may be reached. To complicate matters even further, exact analytical solutions are frequently not available when we attempt to solve problems of reasonable complexity.

Considering a given mixed shell formulation, we can distinguish two ways of checking whether the convergence rate of that formulation can be expected to be uniformly optimal.

The first method consists in carrying out a numerical inf-sup test, which, if passed, assures that the analytical inf-sup condition is satisfied for the given geometry and boundary conditions [3]. Such tests are available for plate problems [10], shell problems [11], incompressible material problems $[2,12]$, contact problems [13]. One difficulty with numerical inf-sup tests is that they are not necessarily equivalent to the corresponding inf-sup condition, i.e. a certain finite element formulation could fail the numerical inf-sup test although it satisfies the inf-sup condition because the numerical test can be more stringent than the inf-sup condition [11] (but let us note that we have not encountered such situation of failing the inf-sup test in actual numerical tests). Also, in some cases, we may accept some locking in a finite element formulation and satisfy ourselves with sub-optimal convergence rates if no better formulation is available.

The second method consists in plotting the convergence to zero of the error norm as meshes are refined for various values of the critical parameter in some selected problems. If these curves have optimal slope and do not shift as the critical parameter is varied, we conclude that uniform optimal convergence is observed. In the case of shell analysis, this may require mesh grading when boundary and internal layers are present in the exact solution. The difficulty with this second method consists in determining and implementing the appropriate norm in which to measure the error. In fact, this norm should be chosen based on the problem under consideration and the mathematical model solved. The choice and implementation of such a norm with emphasis on shell solutions is the topic of the present work.

An outline of the paper is as follows. In Section 2, after a review of convergence measures in displacementbased discretizations, we highlight the difficulties involved in measuring the error in mixed formulations and introduce our proposed norm. Section 3 applies the proposed approach to the MITC family of shell elements [2,14-16]. These elements have been tested in the past using other approaches and are in wide use. The test problems used in the present paper are a hyperboloid of one sheet subjected to a periodic loading, with two different boundary conditions. The choice of these test problems is justified in the paper. Section 4 presents the conclusions of our investigation.

\section{General theory}

In this section, we first recall some results regarding the assessment of the error of displacement-based finite element formulations before considering the problem of assessing the accuracy of mixed-interpolated finite element methods. In Section 2.2, we review some procedures that have been proposed and show their limitations. In Section 2.3, we indicate what properties an ideal error measure would feature and based on those goals we introduce a new error measure in Section 2.4.

\subsection{Displacement-based discretizations}

Consider the very general problem: Find $U \in \mathscr{V}(\mathscr{V}$ being a Hilbert space) such that

$A(U, V)=F(V) \quad \forall V \in \mathscr{V}$

where $A(\cdot, \cdot)$ is a bilinear, continuous and elliptic operator and $F(\cdot)$ is a linear continuous form on $\mathscr{V}$. By application of the Lax-Milgram theorem, the problem in Eq. (1) has a unique solution which satisfies

$\|U\|_{\mathscr{r}} \leqslant c|| F \|_{\mathscr{r}^{\prime}}$

where $c$ is a constant that depends on the material properties involved, thickness of the shell in shell problems, etc., and $\mathscr{V}^{\prime}$ denotes the dual space of $\mathscr{V}$. 
The displacement-based discretization of this problem is: Find $U_{h} \in \mathscr{V}_{h} \subset \mathscr{V}$ ( $\mathscr{V}_{h}$ being the finite element space) such that

$A\left(U_{h}, V_{h}\right)=F\left(V_{h}\right) \quad \forall V_{h} \in \mathscr{V}_{h}$

By application of the Lax-Milgram theorem and Céa's Lemma, we immediately obtain that the solution $U_{h}$ of this problem exists, is unique and satisfies the following bound [2]:

$A\left(U-U_{h}, U-U_{h}\right) \leqslant \inf _{V_{h} \in \mathscr{V}_{h}} A\left(U-V_{h}, U-V_{h}\right)$

which means

$\left\|U-U_{h}\right\|_{\mathscr{V}} \leqslant c d\left(U, \mathscr{V}_{h}\right)$

where $d\left(U, \mathscr{V}_{h}\right)$ is the distance between $U$ and $\mathscr{V}_{h}$ and $c$ is a constant that does not depend on the typical element size $h$ but depends on material properties, shell thickness, etc. Considering the solution of a problem using polynomial shape functions, this leads to the convergence result:

$\left\|U-U_{h}\right\|_{\mathscr{r}} \leqslant c h^{k}$

where $c$ is again a constant that is independent of $h$ but dependent on material properties, shell thickness, etc., and the exact solution $U$, and $k$ is the degree to which the polynomial discretization is complete. Eq. (6) indicates that the best order of convergence we can expect from this discretization is $k$. Of course, the fact that the constant $c$ depends on the material properties, etc. implies that in general the convergence is not uniform in those parameters. For instance, when an almost incompressible material is considered, the constant $c$ can be very large, requiring the use of impractically fine meshes to obtain accurate finite element solutions. Also, within the range of realistic values for $h$, the order $k$ may not be observed. These are manifestations of locking $[1-3,8]$.

Eqs. (1) and (3) imply the important property

$A\left(U-U_{h}, V_{h}\right)=0 \quad \forall V_{h} \in \mathscr{V}_{h}$

which states the orthogonality of the error with respect to the finite element space and implies

$A(U, U)=A\left(U_{h}, U_{h}\right)+A\left(U-U_{h}, U-U_{h}\right)$

which provides an easy way of evaluating the energy of the error $e_{h} \equiv U-U_{h}$ in the finite element solution when the exact solution $U$ is known. Considering the problem of almost incompressible media, using this formula we can readily plot $\log \left(A\left(U-U_{h}, U-U_{h}\right)\right) \equiv \log (A(U, U)-$ $\left.A\left(U_{h}, U_{h}\right)\right)$ versus $\log (h)$ for various values of the Poisson ratio $v$ approaching 0.5 . If a formulation does not lock, curves corresponding to the different values of $v$ essentially coincide and (assuming "ideal" meshing [2]) have a slope of $2 k$. In a formulation that locks, the curves corresponding to different values of $v$ will have a slope lower than $2 k$ or shift—or both —as $v$ is increased to 0.5 .

\subsection{Practical measurement of the error}

The estimation of the difference between the exact solution of a mathematical problem and its approximate numerical solution is a crucial step in an analysis. ${ }^{1}$ In some cases, and in particular if a displacement-based finite element approach is used to reach the numerical solution, it is fairly simple to measure this error: $A\left(U-U_{h}, U-U_{h}\right)$ defines a norm for the error in the displacements, as indicated in Section 2.1. However, when mixed-interpolated formulations are used this approach can not be followed, and there is no wellestablished procedure to measure the error. A variety of techniques have been used by researchers over the years, and we review here some of these methods with emphasis on the analysis of shells.

\subsubsection{Analytical versus numerical reference solutions}

The reference solutions $U$ can either be obtained through analytical methods or numerical methods.

Frequently when analytical methods are used, simplifying assumptions (shallow shell assumption, etc.) must be made to render the problem tractable. These assumptions can result in inconsistencies between the analytical problem and the numerical problem (i.e. as the mesh is refined, the finite element solution does not converge to the reference analytical solution because the reference solution and the finite element formulation are based on fundamentally different mathematical models). This is particularly the case when the finite elements considered are of the degenerated type (such as the MITC elements and other mixed-interpolated formulations) because there are few analytical solutions to meaningful shell problems based on the degenerated approach. Even for simpler shell mathematical models, there are few meaningful complex problems for which closed-form analytical solutions exist at finite thicknesses. Often the analytical solutions that are available are in the form of infinite series (resulting from a Fourier or similar analysis), the coefficients of which usually need to be evaluated numerically.

\footnotetext{
${ }^{1}$ Error measurement procedures, the topic of this paper, are used for a different purpose than error estimators [17-19]. While we set out to evaluate the discrepancy between $U$ and $U_{h}$ when $U$ (or a close approximation thereof) is known, error estimators are used to evaluate the discrepancy between $U$ and $U_{h}$ without any knowledge of $U$. Error estimators are typically used in automatic re-meshing algorithms to determine what regions of a structure require mesh refinement.
} 
In any case, in practice, analytical solutions can be obtained only when very simple geometries are considered.

For these reasons, numerical methods are frequently preferred in obtaining reference solutions. There are of course some pitfalls associated with this approach as well, in particular the fact that the numerical scheme used to establish the reference solution must be consistent with the formulation analyzed. Another issue lies in obtaining a numerical reference solution that is sufficiently accurate. As shown in Ref. [2], if an inappropriate formulation is employed, the number of finite elements needed to obtain a reasonably accurate reference solution can be much larger than it would be if an appropriate scheme were used. In fact, the number of elements needed may be so large that the problem can hardly be tackled with widely available computers. Clearly, we should avoid using such methods whenever possible.

\subsubsection{Pointwise schemes}

Regardless of whether an analytical or numerical reference solution is used, comparing pointwise values of the displacements to a reference solution cannot give a complete measure of the accuracy of the finite element solution. Say for instance that the displacement at one point of a structure calculated using a series of increasingly refined meshes converges to the reference value; there is no guarantee whatsoever that displacements at other points converge in a similar manner since convergence may be non-uniform spatially. Also, the appropriate space for the study of the convergence of shell problems is generally a combination of $H^{1}$ spaces and pointwise values of functions are meaningless in those spaces [8].

Hence only evaluating the error at a point (or a number of points or just a line) cannot be used to rigorously analyze the presence of locking in a finite element shell formulation. That is not to say that evaluating the error at a point is always worthless. Indeed, when an engineer tackles a new problem or tackles a familiar problem with a new type of finite element, such evaluation provides a quick check that a reasonable solution has been attained. However when a deeper understanding or assessment of the performance of a finite element formulation is sought more advanced techniques are clearly necessary.

\subsubsection{A global scheme}

In Ref. [20], the approach used for displacementbased elements was extended to the assessment of the accuracy of mixed-formulated shell finite elements. In this study, the authors plotted " $\ln \left(\mid 1-\left(A^{\mathrm{AS}}\left(U_{h}, U_{h}\right) \mid\right.\right.$ $A(U, U)) \mid)$ " versus " $\ln (h)$ " where $A^{\mathrm{AS}}\left(U_{h}, U_{h}\right)$ denotes the mixed-interpolated form used in the finite element solution.
There are a number of difficulties in extending to mixed formulations an approach originally designed for the assessment of the performance of displacementbased formulations. Namely, in a mixed formulation we have, in general,

$A^{\mathrm{AS}}(U, U)-A^{\mathrm{AS}}\left(U_{h}, U_{h}\right) \neq A^{\mathrm{AS}}\left(U-U_{h}, U-U_{h}\right)$

and $\left(A^{\mathrm{AS}}(U, U)-A^{\mathrm{AS}}\left(U_{h}, U_{h}\right)\right)$ is not a norm for $U-U_{h}$. Hence, there is no guarantee that $\left(A^{\mathrm{AS}}(U, U)-\right.$ $\left.A^{\mathrm{AS}}\left(U_{h}, U_{h}\right)\right)$ is positive, and numerical experiments show that this quantity is frequently negative. This is an immediate result of the energy convergence not necessarily being from below in a mixed formulation. Indeed, it is the fact that $\left(A^{\mathrm{AS}}(U, U)-A^{\mathrm{AS}}\left(U_{h}, U_{h}\right)\right)$ is frequently negative that prompted in Ref. [20] the use of $\ln (\mid 1-$ $\left.\left(A^{\mathrm{AS}}\left(U_{h}, U_{h}\right) / A(U, U)\right) \mid\right)$ rather than $\ln \left(1-\left(A^{\mathrm{AS}}\left(U_{h}, U_{h}\right) /\right.\right.$ $A(U, U)))$.

Hence, the approach of Ref. [20], although an improvement over measuring differences in solutions at a point only, may not be sufficiently stringent to assess whether a mixed finite element scheme is optimal.

\subsection{Design of an error measure}

From the previous section, it is clear that the proper procedure to assess the performance of mixed-interpolated shell finite elements should ideally satisfy the following conditions:

1. It should take into account the finite element solution-and of course the exact solution-over the entire structure, not just over a few selected points or lines.

2. It should give quantitative results regarding the performance of the element tested. In particular, it should define a proper norm for the error. Hence, we denote this error measure by $\left\|U-U_{h}\right\|_{*}$ where $U$ denotes the exact solution and $U_{h}$ denotes a finite element solution. The norm should be easily related to the physical problem analyzed (i.e. we would like a physics-based norm).

3. We should be able to employ the procedure whether or not an analytical solution is available for the problems considered. Indeed the procedure should be flexible enough that it can handle arbitrary geometries, loadings, etc.

4. It should be possible to employ a single procedure to assess the performance of various element formulations, allowing easy comparison between different formulations.

5. A method that can be easily related to the assessment method already existing for displacement-based formulations is highly desirable. Of course a procedure that can be easily extended to mixed finite element formulations other than shell finite elements - such 
as $\mathrm{u} / \mathrm{p}$ formulations for the analysis of incompressible materials and incompressible fluids-would also be of great value.

6. Finally, in the case of shell structures, the same norm would be used for all shell problems, regardless of their asymptotic behaviors and regardless of in which norm the asymptotic behavior is measured.

Given such an error measure, the goal in developing shell finite elements would be to identify elements such that, for any problem with a relative thickness $\epsilon=t / L$ considered, the finite element solution $U_{h}^{\epsilon}$ and the reference solution $U_{\text {ref }}^{\epsilon}$ satisfy

$\frac{\left\|U_{\mathrm{ref}}^{\epsilon}-U_{h}^{\epsilon}\right\|_{*}}{\left\|U_{\mathrm{ref}}^{\epsilon}\right\|_{*}}=C h^{k}$

for all thicknesses, with $C$, a constant independent of the shell thickness (and of course element size $h$ ), and $k$, the order of the complete polynomial included in the formulation (see [8]). Such a formulation would be uniformly optimal and would be guaranteed not to lock.

Based on these objectives, we propose a new procedure in Section 2.4.

\subsection{Proposed error measure}

We present our error measure in the context of the analysis of shells. In order to make our presentation of the proposed algorithm simple, we first assume that an analytical solution of the shell problem considered is available. This requirement will be removed later on.

So for now, let us assume that we are considering a shell problem based on the Reissner-Mindlin kinematic assumption (see $[8,21,22])$ for which we know the exact analytical solution for the displacements and rotations, collectively denoted by $U$. For this problem we can therefore evaluate the exact strains at every point $x$ in the geometry. We denote the shell domain by $\Omega$ and the exact Cartesian strains by $B(x) U(x)$ where $B(x)$ is the strain operator. We discretize this problem and solve it using a certain finite element formulation, yielding a finite element solution $U_{h}\left(x_{h}\right)$ (again, $U_{h}$ denotes collectively translations and rotations) at every point of the discretized domain $\Omega_{h}$. Our finite element formulation allows us to calculate the mixed finite element Cartesian strains $B_{h}\left(x_{h}\right) U_{h}\left(x_{h}\right)$ related to this solution at every point $x_{h} \in \Omega_{h}$, where $\Omega_{h}$ denotes the computational domain.

Since we have discretized the geometry, the finite element domain and the actual domain are different in general, see Fig. 1.

It is however possible to define a one-to-one mapping between every point $x_{h} \in \Omega_{h}$ and every point $x \in \Omega$. Let us denote this mapping by $\Pi$, so that

$\forall x \in \Omega \quad \exists ! x_{h} \in \Omega_{h} \quad$ such that $x=\Pi x_{h}$

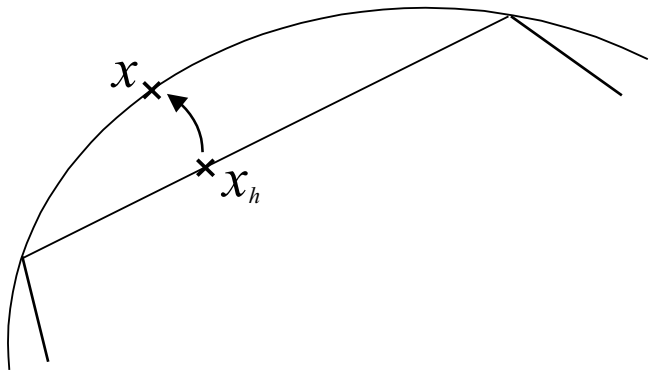

Fig. 1. Mapping between a coarse mesh and the exact geometry. The mapping defines a bijection between the coarse mesh (straight line) and the exact geometry of the structure (curved line).

It should be noted that there is not a unique way of selecting $\Pi$ : proper care should therefore be taken to select a reasonable mapping.

The error measure EM we propose is then simply

$\operatorname{EM}\left(U, U_{h}\right)=\frac{1}{2} \int_{\Omega} \Delta e^{\mathrm{T}} C(x) \Delta e \mathrm{~d} \Omega$

where $C(x)$ denotes the material law expressed in the Cartesian coordinate system and

$\Delta e=B(x) U(x)-B_{h}\left(\Pi^{-1}(x)\right) U_{h}\left(\Pi^{-1}(x)\right)$

In physical terms, $\Delta e$ denotes the difference between the exact strains at one point of the structure and the finite element strains at the corresponding point of the mesh, while $\operatorname{EM}\left(U, U_{h}\right)$ is simply the strain energy associated with this difference. EM is the square of a norm for $\Delta e$. It is however not the square of a norm for the error in the displacements.

Note that if we were interested in defining an error measure that is also a norm for the error in the displacements we may consider using as an alternative error measure $\mathrm{EM}^{\prime}$ defined by $\operatorname{EM}^{\prime}\left(U, U_{h}\right)=\operatorname{EM}\left(U, U_{h}\right)+$ $C|| u-u_{h}\left\|_{L^{2}}^{2}+C^{\prime}|| \theta-\theta_{h}\right\|_{L^{2}}^{2}$ where $C$ and $C^{\prime}$ are constants needed for the sake of dimensional homogeneity; $u$ denotes the exact translations and $u_{h}$ denotes the finite element translations. These constants would need to be selected carefully to assure that $\mathrm{EM}^{\prime}$ can be used to detect locking. Also, in practice, these constants would need to be selected based on typical dimensions, material properties, etc. of the problem in such a way as to assure that in numerical experiments $\operatorname{EM}\left(U, U_{h}\right)$ and $C \| u-$ $u_{h} \|_{L^{2}}^{2}$ and $C^{\prime}\left\|\theta-\theta_{h}\right\|_{L^{2}}^{2}$ are of comparable magnitudes (see Appendix A).

In case an analytical solution is not available for the problem considered, we can substitute in Eq. (12) an accurate approximation for the strains of the exact solution. This accurate approximation can be obtained for example by using a finite element method with a very fine mesh. Note that it is entirely possible to employ 
finite elements of a different type than the element analyzed to obtain this reference solution, provided the finite element scheme used to establish the reference solution is consistent with the mathematical model employed.

In rigorous terms, consider the mapping $\Pi$ between the coarse mesh domain $\Omega_{h}$ and the reference fine mesh domain $\Omega_{\text {ref }}$ (see Fig. 2). Then we use as our error estimate

$\operatorname{EM}\left(U, U_{h}\right)=\frac{1}{2} \int_{\Omega_{\mathrm{ref}}} \Delta e^{\mathrm{T}} C(x) \Delta e \mathrm{~d} \Omega_{\mathrm{ref}}$

where

$\Delta e=B_{\text {ref }}(x) U_{\text {ref }}(x)-B_{h}\left(\Pi^{-1}(x)\right) U_{h}\left(\Pi^{-1}(x)\right)$

To analyze convergence rates and locking, we propose to plot $\ln \left(\operatorname{EM}\left(U, U_{h}\right) / E(U, U)\right)$ versus $\ln (h)$ with the notation $E(U, U) \equiv \frac{1}{2} A(U, U)$. In practice $A(U, U)$ may be replaced with the energy of the numerically computed reference solution $A^{\mathrm{AS}}\left(U_{\text {ref }}, U_{\text {ref }}\right)$.

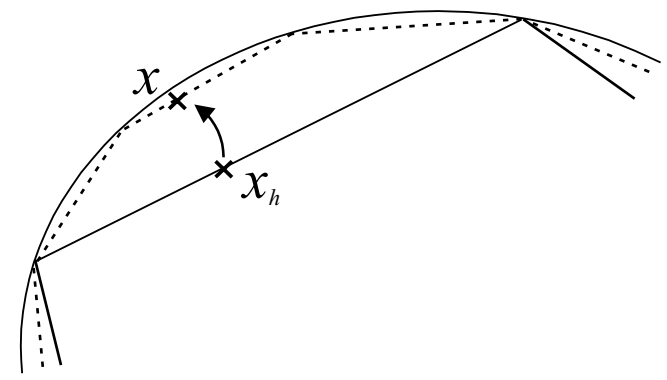

Fig. 2. Mapping between a coarse mesh and a reference mesh. The mapping defines a bijection between the coarse mesh (solid straight line) and the reference mesh (dotted straight line). The exact geometry is also shown (curved line).
It is important to observe that when the geometry of the problem considered allows for $\Pi$ to be the identity operator (i.e. the geometry is exactly represented) and a displacement-based formulation is analyzed then clearly we have $\operatorname{EM}\left(U, U_{h}\right)=E(U, U)-E\left(U_{h}, U_{h}\right)=$ $E\left(U-U_{h}, U-U_{h}\right)$. Therefore in that case plotting $\ln \left(\operatorname{EM}\left(U, U_{h}\right) / E(U, U)\right)$ is equivalent to plotting $\ln \left(1-A\left(U_{h}, U_{h}\right) / A(U, U)\right)$. Hence in this case the proposed procedure reduces to the usual procedure employed to analyze the convergence rate of a displacement-based formulation.

\section{Numerical experiments: MITC shell elements}

In this section, we introduce two test problems for the assessment of the performance of shell finite elements. We then apply the error measure introduced in Section 2.4 to these problems.

\subsection{Description of the test problems}

The two test problems use the same geometry given in Fig. 3 and the same loading.

The mid-surface of this structure is described by the equation

$x^{2}+z^{2}=1+y^{2}$

i.e. the mid-surface is a hyperboloid of one sheet, a doubly ruled surface. The loading imposed is the periodic pressure

$p(\theta)=P_{0} \cos (2 \theta)$

where $\theta$ denotes the polar angle, as shown in Fig. 3. Using symmetries, the analyses were performed using one eighth of the structure. Note that the analyses can
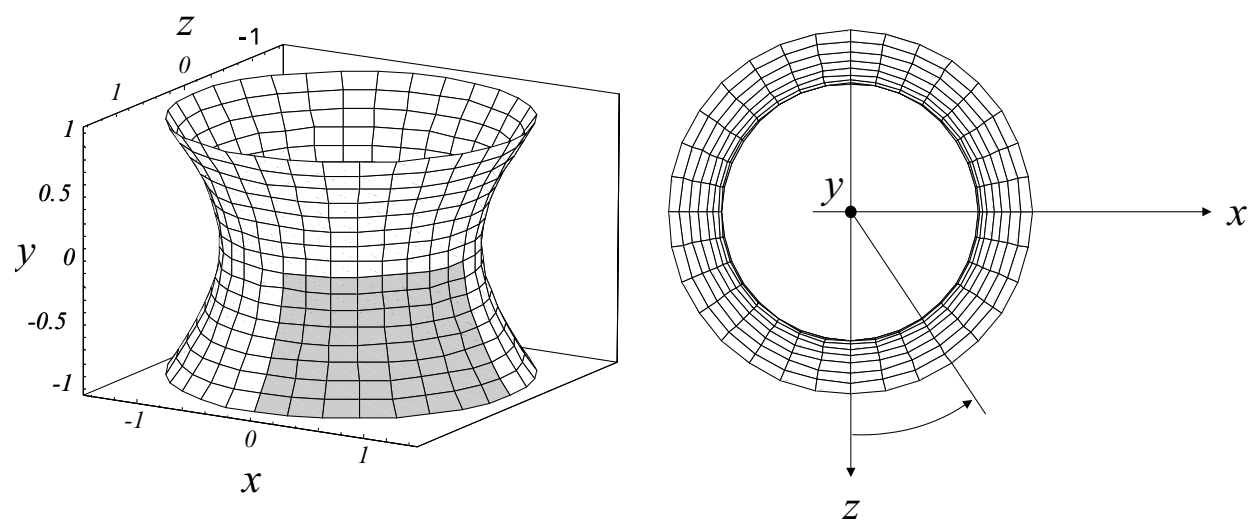

Fig. 3. Hyperboloid's mid-surface geometry. The polar angle $\theta$ used to describe the loading is indicated. Also $-1 \leqslant y \leqslant 1$ (Young's modulus $=2.0 \mathrm{E} 11$, Poisson's ratio $=1 / 3$ ). 
also be carried out on one sixteenth of the structure using antisymmetry conditions at $\theta=45^{\circ}$.

\subsubsection{Clamped hyperboloid}

In the first test problem we impose clamped boundary conditions at both ends $y=1$ and -1 . This problem is similar to the clamped cylinder problem which is frequently used to test a shell finite element formulation. That problem is well known to be membrane-dominated and to feature boundary layers in the regions near the clamped boundaries. We use this new test because unlike the clamped cylinder problem, the hyperboloid problem has non-zero Gaussian curvature, making it a more general and also a tougher problem to solve. Indeed the geometry of the mid-surface does not lead to aligning the sides of quadrilateral elements with the asymptotic lines. It is known that finite elements perform better when their sides are oriented that way (see $[4,8])$. In industrial applications though it is generally not possible to align the mesh with the asymptotic lines and therefore we should not use such alignment in our tests.

The first step with a new problem like this one consists in determining the asymptotic behavior. We follow here the method presented in Ref. [7]: we study the behavior of $\bar{\rho}$ defined as

$\bar{\rho}=\frac{\log \left(E_{h}\left(t_{i+1}\right)\right)-\log \left(E_{h}\left(t_{i}\right)\right)}{\log \left(t_{i}\right)-\log \left(t_{i+1}\right)}$

where $E_{h}\left(t_{i}\right)$ denotes the finite element strain energy with the thickness $t_{i}$. For our study, we use the sequence of thicknesses $t_{i}=10^{-i}$ with $i \in\{2,3,4,5,6\} .{ }^{2}$ For each thickness, the problem is solved using a mesh consisting of $72 \times 72$ MITC16 shell elements. A boundary layer is present along the clamped edge, see Fig. 4. Due to the presence of this layer, we need to use locally refined meshes (also referred to as "graded meshes") to properly identify the element convergence behaviors (see Section 3.3).

In our mesh grading scheme, the modelled geometry is subdivided into two regions: a band of width $6 \sqrt{t_{i}}$ along the clamped boundary, and the remainder of the geometry. Each of these two regions is then meshed using a uniform mesh (see Fig. 5). We used the same number of elements in each of the two regions. Note that the width $6 \sqrt{t_{i}}$ is selected based on the theoretical knowledge that along a line that is not an asymptotic line, layer thicknesses vary as $\sqrt{t}$ [23], and the constant 6 is chosen based on numerical experiments. For each thickness, the smoothness of the numerical solution is checked. The results, obtained with $72 \times 72$ element

\footnotetext{
${ }^{2}$ Of course, not all these thicknesses correspond to realistic physical problems.
}

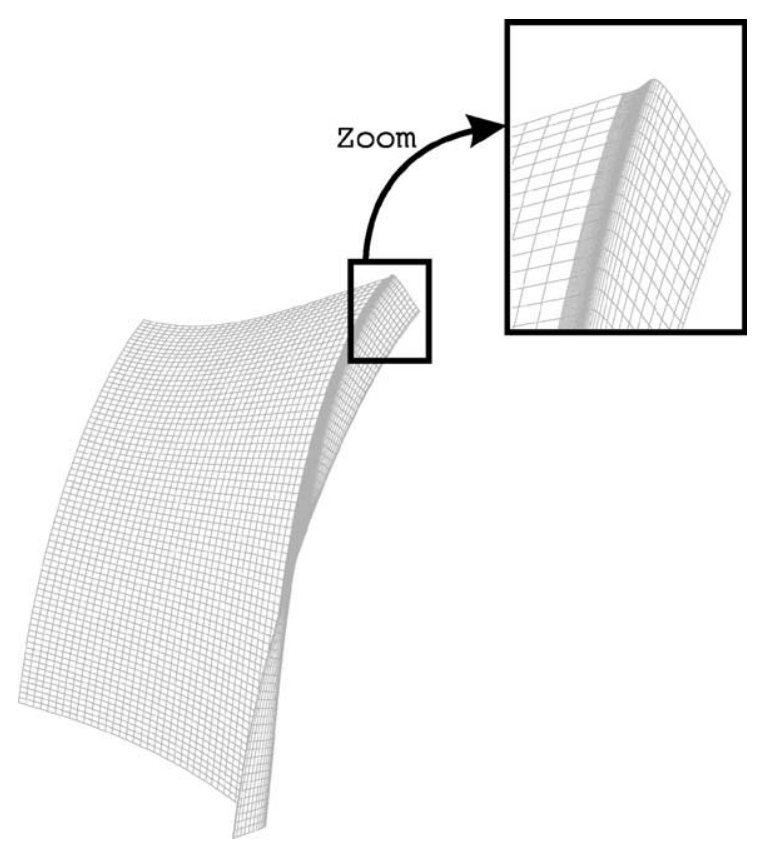

Fig. 4. Deformation of the clamped hyperboloid. The boundary layer along the clamped edge is clearly visible. Graded $72 \times 72$ MITC16 mesh, $t=0.0001, L=1$.

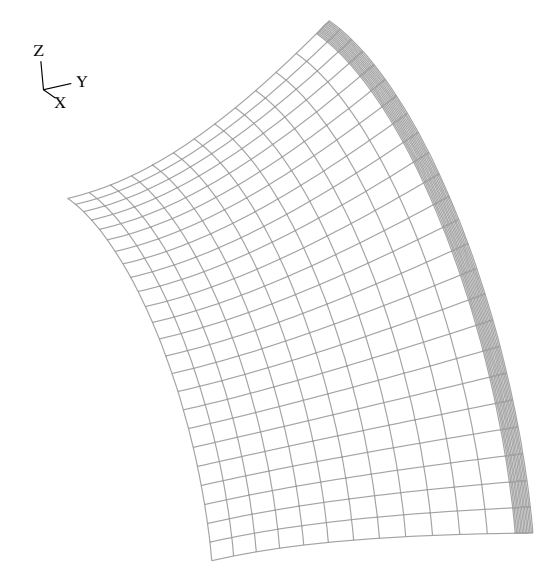

Fig. 5. Hyperboloid: example of a $24 \times 24$ graded mesh. There are $12 \times 24$ elements in the region near the boundary and $12 \times 24$ elements in the rest of the mesh.

MITC16 meshes and presented in Table 1 show that this problem is membrane-dominated (this is, $\bar{\rho}$ approaches 1) as the thickness is decreased.

\subsubsection{Free hyperboloid}

In the second test problem the formerly clamped edges are left free.

This problem is similar to the free cylinder problem which is frequently used to test a shell finite element 
Table 1

Asymptotic behavior of the clamped hyperboloid problem

\begin{tabular}{lll}
\hline Thickness & Strain energy & $\bar{\rho}$ \\
\hline $10^{-2}$ & $0.539187 \times 10^{3}$ & 1.0465 \\
$10^{-3}$ & $0.600115 \times 10^{4}$ & 1.0134 \\
$10^{-4}$ & $0.618988 \times 10^{5}$ & 1.0041 \\
$10^{-5}$ & $0.624889 \times 10^{6}$ & 1.0013 \\
$10^{-6}$ & $0.626748 \times 10^{7}$ & \\
\hline
\end{tabular}

formulation. That problem is known to be bendingdominated.

Similarly to the clamped hyperboloid, we use this new test because unlike the free cylinder problem, the free hyperboloid problem has non-zero Gaussian curvature, making it a more general and also a tougher problem to solve. In this case we use the boundary layer width $=0.5 \sqrt{t_{i}}$.

The asymptotic behavior of this problem is again determined by employing the approach presented in Ref. [7]. The results given in Table 2 show that the free hyperboloid problem is bending-dominated.

\subsection{Consistency results}

If a finite element formulation is consistent with the basic shell model of Refs. [8,24] then necessarily a very fine mesh of elements will give a strain energy close to the exact solution of the shell model. The difficulty in

Table 2

Asymptotic behavior of the free hyperboloid problem

\begin{tabular}{lll}
\hline Thickness & Strain energy & $\bar{\rho}$ \\
\hline $10^{-2}$ & $0.452847 \times 10^{6}$ & 2.9959 \\
$10^{-3}$ & $0.448612 \times 10^{9}$ & 2.9999 \\
$10^{-4}$ & $0.448552 \times 10^{12}$ & 3.0000 \\
$10^{-5}$ & $0.448502 \times 10^{15}$ & \\
\hline
\end{tabular}

using this property to test for consistency-besides the fact that it is only a necessary condition for consistency - is that while we need to prove consistency for complex geometries and loading conditions, usually analytical solutions for such problems are not available and therefore we do not know the strain energy of the exact solution. To generate a reference strain energy we can however make use of the fact that for a displacement-based formulation, we have

$E_{h}-E_{0} \simeq C h^{2 k}$

where $E_{h}$ is the strain energy of the finite element solution obtained using elements of typical size $h, E_{0}$ is the strain energy of the exact solution and $k$ is the degree of the complete polynomial included in the formulation (see [2]). We can make use of Eq. (19) to evaluate $E_{0}$ from the strain energies $E_{h 1}$ and $E_{h 2}$ in two finite element models with typical element sizes $h_{1}$ and $h_{2}$. In Table 3, we report the reference energy extrapolated by this method with the use of $24 \times 24$ and $72 \times 72$ displacement-based 16-node element meshes of the clamped hyperboloid problem (graded meshes are employed). This same table also reports the strain energies obtained with fine meshes of MITC4, MITC9 and MITC16 elements. For the MITC4 (resp. MITC9, MITC16) element, we use a $192 \times 192$ (resp. $96 \times 96,72 \times 72$ ) element graded mesh. The strain energies obtained with the MITC elements, considering $t$ as small as $10^{-5}$, agree well with the extrapolated reference strain energies.

In Table 4, we report the results obtained for the free hyperboloid, and again we observe good agreement between the strain energies of the MITC finite element solutions and reference strain energies obtained by extrapolation.

As a more refined approach, we now make use of our error measure by evaluating in Eq. (14) the reference strain $e_{\text {ref }}=B_{\text {ref }} U_{\text {ref }}$ by use of a very fine mesh of displacement-based elements (we use the fine meshes introduced above) and $e_{h}=B_{h} U_{h}$ with a number of coarse meshes made of MITC elements. If the MITC elements yield a solution that converges to the solution of the basic shell model of Refs. [8,24], then the error measure must go to zero as the MITC mesh is refined. Obviously, we would also like to observe the error measure con-

Table 3

Strain energy for clamped hyperboloid problem: consistency

\begin{tabular}{lllll}
\hline$t$ & $\begin{array}{l}\text { Extrapolated value using 16-node } \\
\text { displacement-based elements }\end{array}$ & MITC4 & MITC9 & MITC16 \\
\hline $10^{-2}$ & $0.539187 \times 10^{3}$ & $0.539136 \times 10^{3}$ & $0.539187 \times 10^{3}$ & $0.539187 \times 10^{3}$ \\
$10^{-3}$ & $0.600115 \times 10^{4}$ & $0.600030 \times 10^{4}$ & $0.600115 \times 10^{4}$ & $0.600115 \times 10^{4}$ \\
$10^{-4}$ & $0.618988 \times 10^{5}$ & $0.618787 \times 10^{5}$ & $0.618988 \times 10^{5}$ & $0.618988 \times 10^{5}$ \\
$10^{-5}$ & $0.624889 \times 10^{6}$ & $0.624365 \times 10^{6}$ & $0.624889 \times 10^{6}$ & $0.624889 \times 10^{6}$ \\
$10^{-6}$ & $0.627608 \times 10^{7}$ & $0.625663 \times 10^{7}$ & $0.626748 \times 10^{7}$ & $0.626748 \times 10^{7}$ \\
\hline
\end{tabular}


Table 4

Strain energy for free hyperboloid problem: consistency

\begin{tabular}{|c|c|c|c|c|}
\hline$t$ & $\begin{array}{l}\text { Extrapolated value using 16-node } \\
\text { displacement-based elements }\end{array}$ & MITC4 & MITC9 & MITC16 \\
\hline $10^{-2}$ & $0.452847 \times 10^{6}$ & $0.452847 \times 10^{6}$ & $0.452847 \times 10^{6}$ & $0.452847 \times 10^{6}$ \\
\hline $10^{-3}$ & $0.448610 \times 10^{9}$ & $0.448609 \times 10^{9}$ & $0.448610 \times 10^{9}$ & $0.448612 \times 10^{9}$ \\
\hline $10^{-4}$ & $0.448620 \times 10^{12}$ & $0.448845 \times 10^{12}$ & $0.448747 \times 10^{12}$ & $0.448552 \times 10^{12}$ \\
\hline
\end{tabular}

verge with the optimal convergence order allowed by the interpolation orders of each element. The numerical results for the clamped hyperboloid are shown in Fig. 6 where the relative consistency measure $\operatorname{CM}\left(U_{\text {ref }}, U_{h}\right)=$ $\operatorname{EM}\left(U_{\text {ref }}, U_{h}\right) / E\left(U_{\text {ref }}, U_{\text {ref }}\right)$ is plotted versus the typical element size $h$. Note that a sufficiently accurate solution could not be obtained with the displacement-based 4node element in this problem. Fig. 6 shows that the optimal order of convergence is essentially observed for
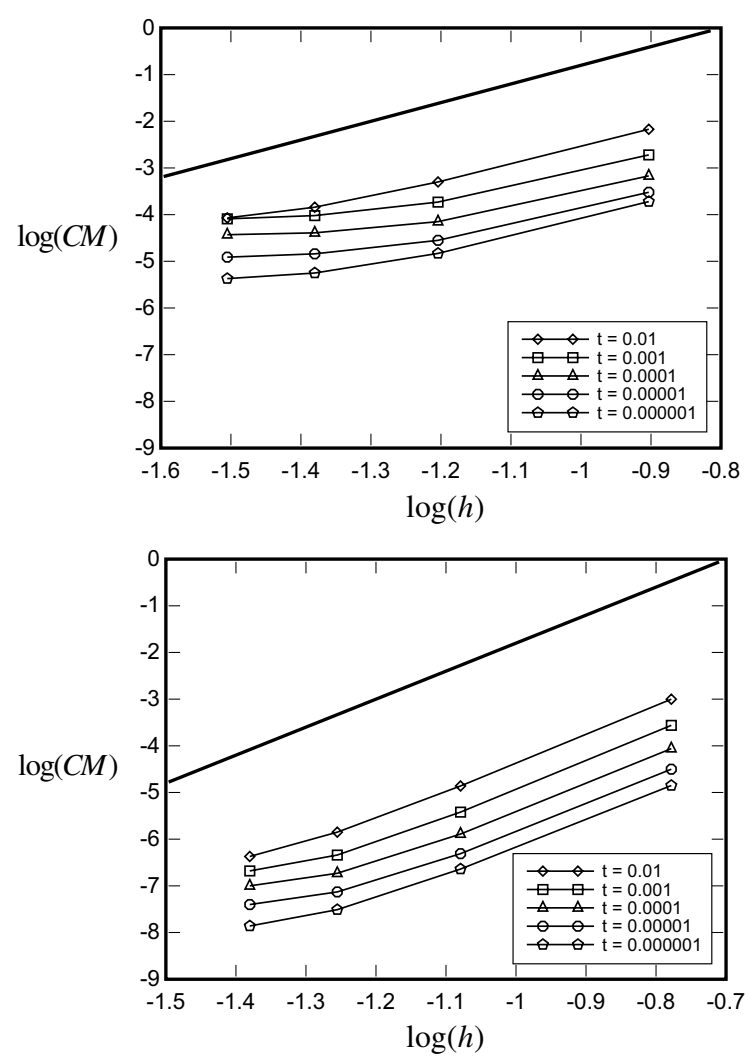

Fig. 6. Clamped hyperboloid: consistency. Graded meshes are employed. Top to bottom: MITC9, MITC16. For 4-node elements accurate enough reference solutions could not be reached with the displacement-based element. $\mathrm{CM}$ denotes the relative convergence measure, i.e. $\mathrm{CM}=\left(\int_{\Omega} \Delta e^{\mathrm{T}} C \Delta e \mathrm{~d} \Omega\right) / E\left(U_{\text {ref }}, U_{\text {ref }}\right)$. The reference solutions are established with displacement-based 9-node and 16-node elements. The bold lines show the optimal slopes. the two elements considered since all curves have essentially the optimal slope. We notice some tapering of the curves as the typical element size $h$ goes to zero. This is probably due to the fact that our reference solutions are obtained numerically and do not coincide perfectly with the exact solution of the basic shell model.

\subsection{Convergence results}

In this section, we use the error measure presented in Section 2.4 to assess the convergence of the quadrilateral MITC elements.

Since we have established through our numerical experiments of Section 3.2 that the MITC elements are consistent with the mathematical model, we can use these elements to establish our reference solutions. This is particularly important for two reasons. First, we have mentioned in the consistency experiments of Section 3.2 that it was not possible to obtain a very accurate reference solution using the displacement-based 4-node element for the clamped hyperboloid problem. Second, considering now the free hyperboloid, we know that we must expect severe locking due to the bendingdominated nature of the problem if we use the displacement-based elements for this analysis. Lower order displacement-based elements can not be used to establish sufficiently accurate reference strains in this case.

For the two problems, and each of the three MITC elements, we employ a highly refined MITC element mesh to establish a reference solution and coarse meshes to assess the convergence. For the MITC4 element, the coarse meshes consist of $N \times N=24 \times 24,32 \times 32$, $48 \times 48$ and $64 \times 64$ elements, and the reference solution is established with a $192 \times 192$ element mesh. For the MITC9 element, the coarse meshes consist of $8 \times 8$, $16 \times 16,24 \times 24$ and $32 \times 32$ elements, and the reference solution is established with a $96 \times 96$ element mesh. For the MITC16 element, the coarse meshes consist of $6 \times 6$, $12 \times 12,18 \times 18$ and $24 \times 24$ elements, and the reference solution is established with a $72 \times 72$ element mesh.

For each problem and each element, we employ two separate meshes: a uniform mesh (see Fig. 7), and a locally refined mesh, where again we use the refinement method presented in Fig. 5 with the width of the refined regions equal to $6 \sqrt{t}$ (for the clamped condition) and $0.5 \sqrt{t}$ (for the free condition). 


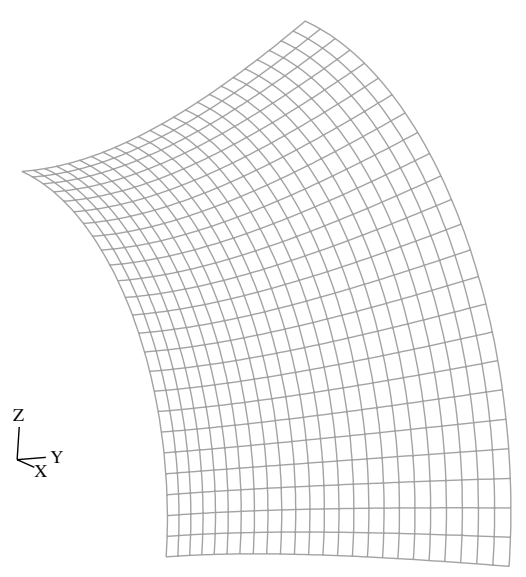

Fig. 7. Hyperboloid: example of a uniform $24 \times 24$ mesh.

In each case, we plot the relative error measure $\operatorname{RE}\left(U_{\text {ref }}, U_{h}\right)=\operatorname{EM}\left(U_{\text {ref }}, U_{h}\right) / E\left(U_{\text {ref }}, U_{\text {ref }}\right)$ against the typical element size $h=1 / N$ as the mesh is refined for the thicknesses $t=10^{-i}$ with $i>1 .^{3}$ If an element provides uniform optimal convergence, the curves corresponding to the different thicknesses will all be parallel to each other and have the optimal slope permitted by the order of their interpolation spaces and there would be little to no upwards vertical shifting of the curves as the thickness is reduced (see Eq. (10)). If the curves do not have the optimal slope, we conclude that the order of convergence is not optimal. If there is some upwards shifting of the curves, we conclude that the convergence is not uniform.

The fact that the meshes are not aligned with the asymptotic lines, the anticlastic nature of the geometry of the mid-surface and the presence of boundary layers combine to make these valuable and quite severe test problems.

\subsubsection{Clamped hyperboloid}

The relative errors RE for the clamped hyperboloid problem using uniform meshes are shown in Fig. 8. We observe that for all three elements studied the order of convergence becomes lower (the curves "flatten out") as the shell thickness is reduced and there is some upwards shifting.

The relative errors RE obtained for the same problem modelled with locally refined meshes are shown in Fig. 9. We observe that for all three elements studied the convergence order remains constant and optimal as the shell thickness is reduced and for the MITC9 and

\footnotetext{
${ }^{3}$ Of course below a certain thickness it is not possible to solve the finite element problem as the stiffness matrices become ill-conditioned, leading to instabilities in the numerical solution or solver failure. We report here all results that could be obtained without ill-conditioning occurring.
}
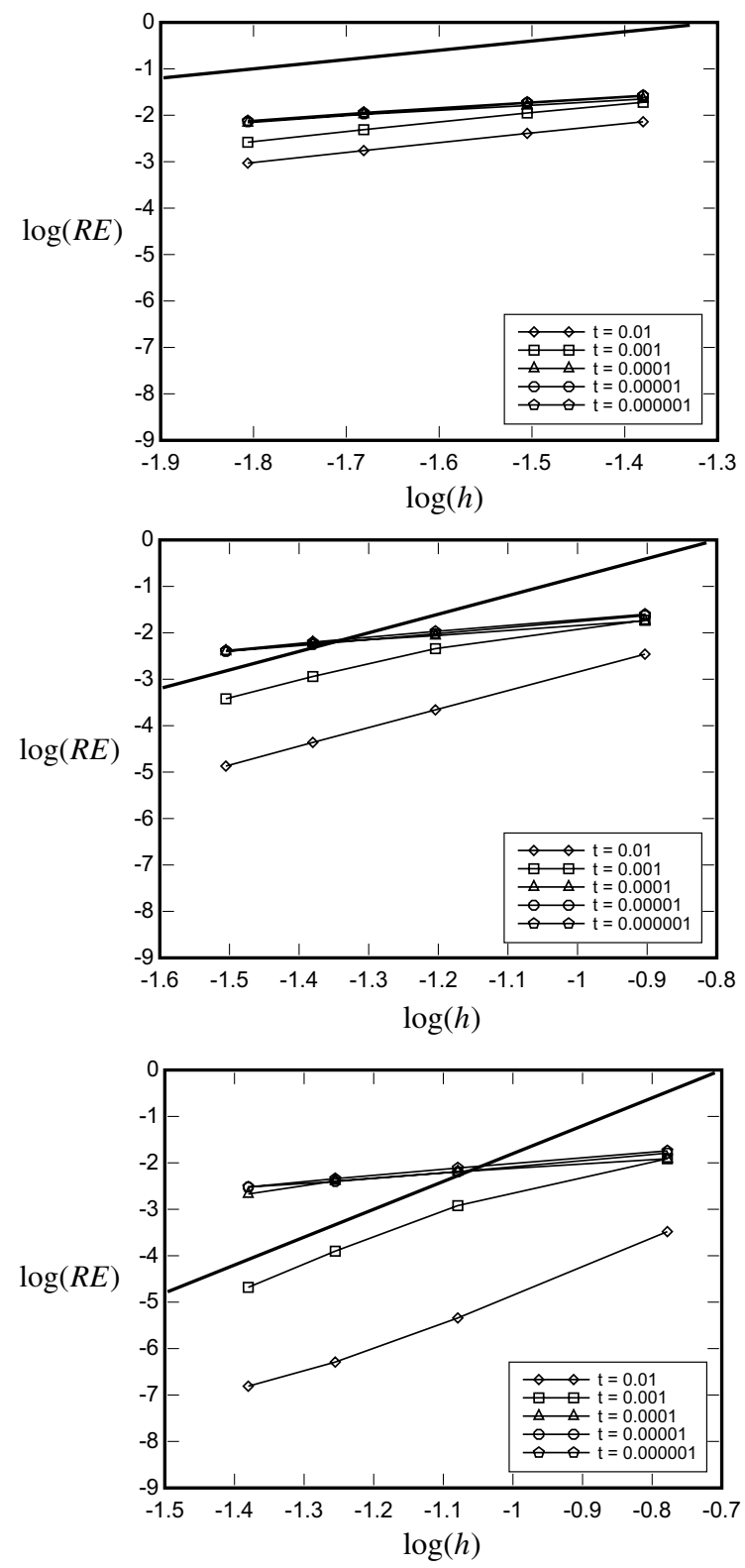

Fig. 8. Clamped hyperboloid modelled with uniform meshes: convergence. Top to bottom: MITC4, MITC9, MITC16. The reference solutions are established using very fine meshes of the same MITC elements. The bold lines show the optimal slopes.

MITC16 there is some minor downwards shifting of the curves indicating that the elements actually perform slightly better at smaller thicknesses. The MITC4 element shows some minor upwards shifting (considering the practical cases $t \geqslant 10^{-4}$ ).

\subsubsection{Free hyperboloid}

The relative errors RE for the free hyperboloid using uniform meshes are shown in Fig. 10. The relative errors 

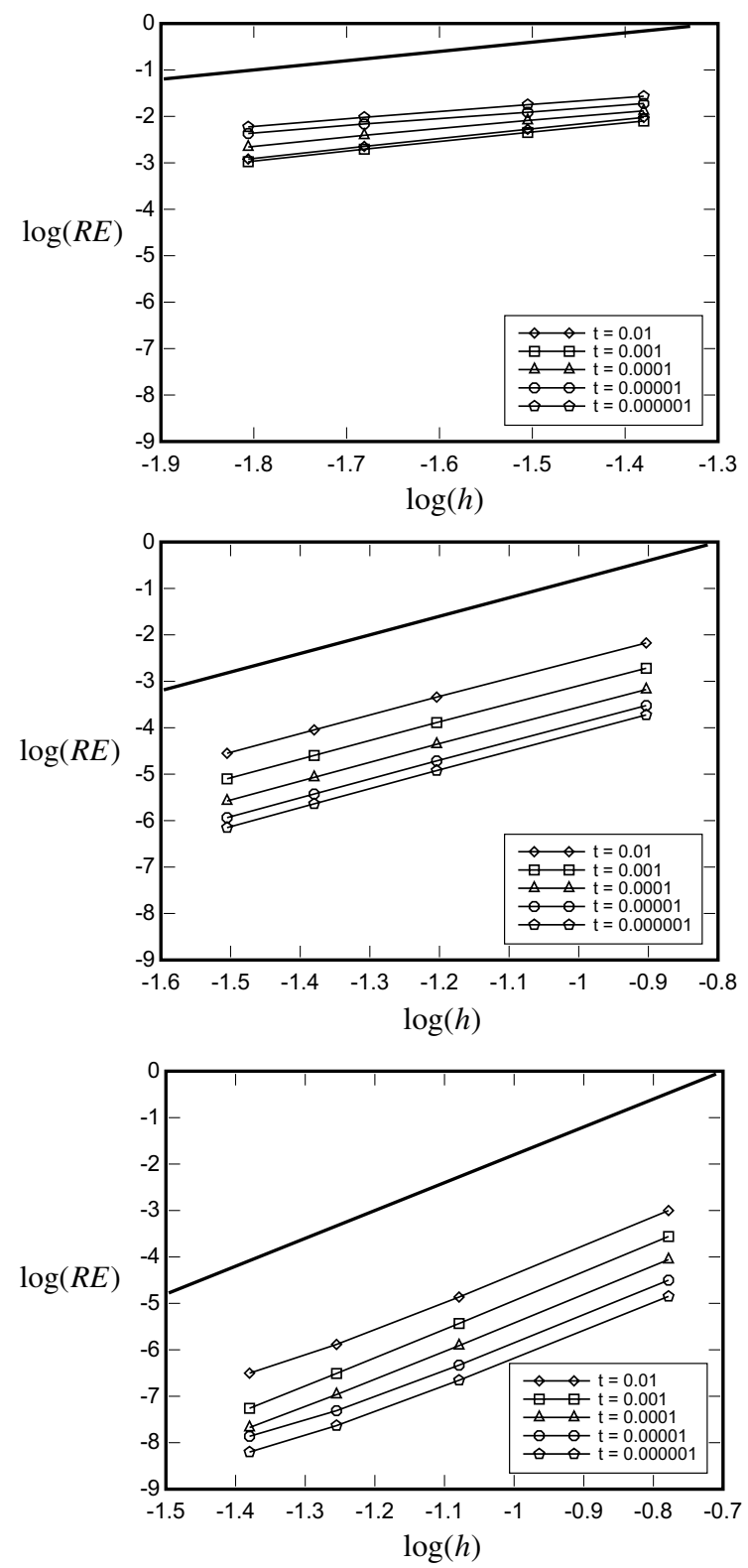

Fig. 9. Clamped hyperboloid modelled with graded meshes: convergence. Top to bottom: MITC4, MITC9, MITC16. The reference solutions are established using very fine meshes of the same MITC elements. The bold lines show the optimal slopes.

RE for the same problem solved using locally refined meshes are shown in Fig. 11.

From Fig. 10, we observe that when uniform meshes are employed for this problem the convergence order is low and clearly sub-optimal for relatively thick shells. When locally refined meshes are employed, we conclude from Fig. 11 that good orders of convergence are re-
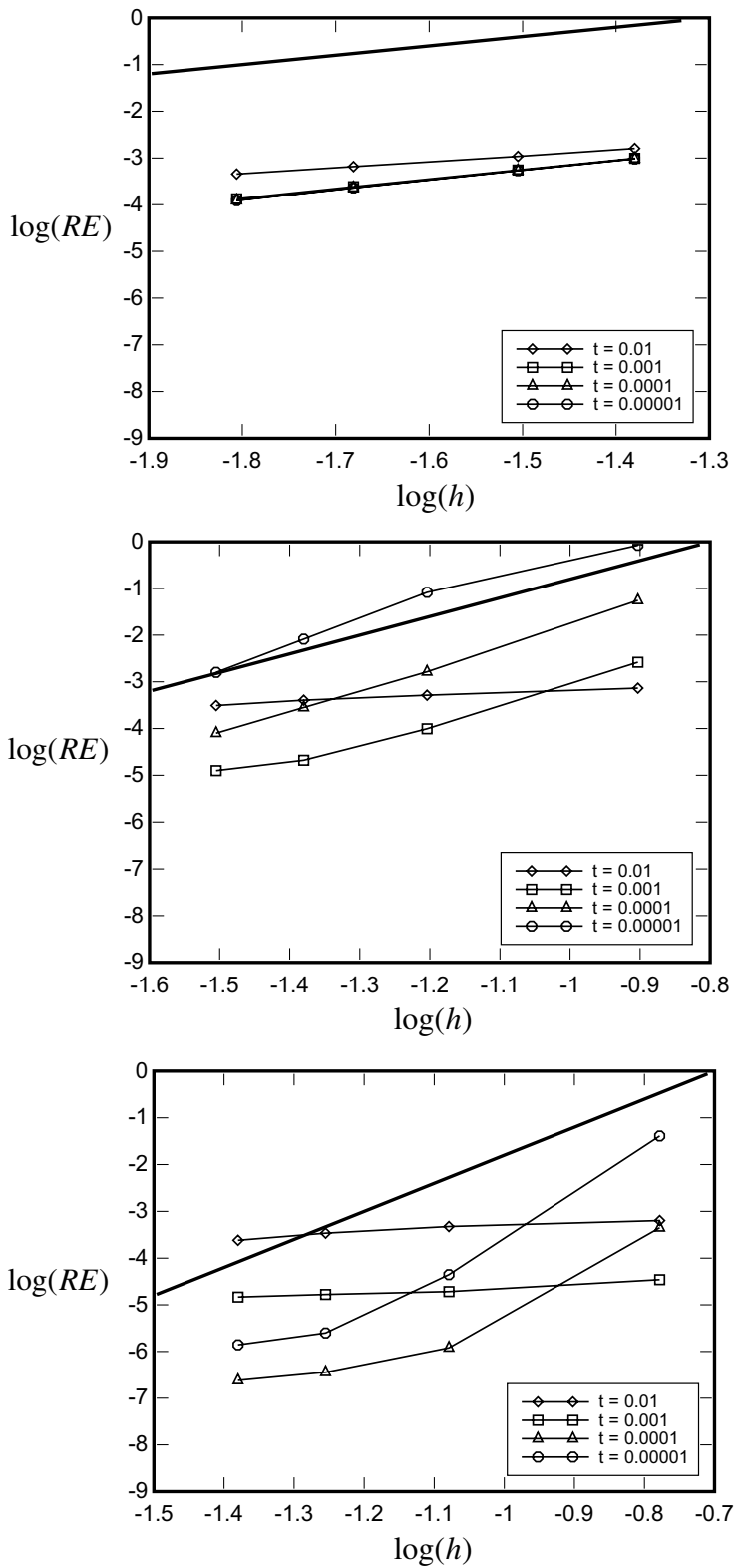

Fig. 10. Free hyperboloid modelled with uniform meshes: convergence. Top to bottom: MITC4, MITC9, MITC16. The reference solutions are established using a very fine mesh of the same MITC elements. The bold lines show the optimal slopes.

covered for all elements. However, in particular for the MITC9 element an upwards shifting of the curves is observed, indicating that the convergence is not uniform. We attribute this non-uniformity to some spurious shear strains in the finite element solution (see Ref. [25]).

These studies clearly show that graded meshes must be used to evaluate shell finite element formulations. 

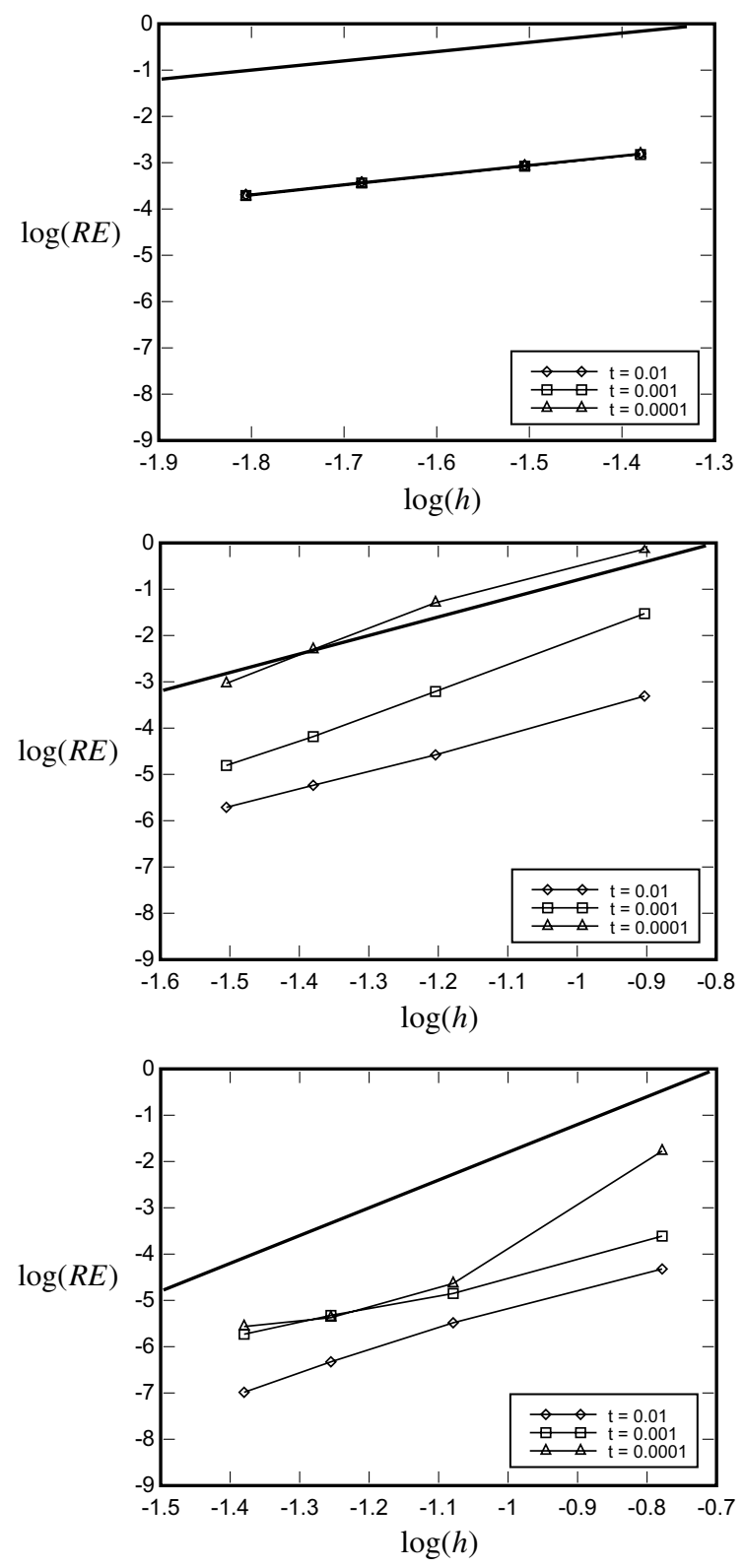

Fig. 11. Free hyperboloid modelled with graded meshes: convergence. Top to bottom: MITC4, MITC9, MITC16. The reference solutions are established using very fine meshes of the same MITC elements. The bold lines show the optimal slopes.

\section{Concluding remarks}

In this paper, we reviewed error measure procedures that have been employed to test existing mixed-formulated finite element schemes. In particular, we indicated that there is a need for a procedure that allows to rigorously assess the discrepancy between the exact mathematical solution of a shell problem and the approximate solution of the same problem obtained through the use of a finite element procedure. We designed a new error measure method that can be used to evaluate this discrepancy. Our proposed error measure, which is physically based, defines a norm for the error in the strains and can be extended to other problems where mixedinterpolated finite element methods are used, such as plates, beams or incompressible materials.

We used this error measure to numerically analyze the quadrilateral MITC shell elements with two severe test problems. First we confirmed that the elements are consistent with the basic shell mathematical model of Refs. [8,24]. Then we studied the convergence properties of the MITC elements. Specifically, we investigated the influence of using uniform versus graded meshes on the convergence rates observed in a membrane-dominated problem and a bending-dominated problem. Our investigation of the MITC elements indicates that the use of locally refined meshes in the boundary layers is crucial in allowing good convergence rates to be observed. In the case of the membrane-dominated problem, uniformly optimal convergence is observed. In the case of the bending-dominated problem, the MITC4 element performs extremely well, and also the MITC9 and MITC16 show good orders of convergence. However, the convergence is not uniform for the MITC16 element and specifically for the MITC9 element, which therefore should be improved [25].

A natural extension of the work presented here would be to study, using the same approach, other existing mixed finite element formulations, such as the isoparametric $\mathrm{u} / \mathrm{p}$ elements used in incompressible material analysis, the MITC plate elements and the many mixed-formulated shell elements that have been proposed in recent years.

\section{Acknowledgements}

We would like to express our gratitude for the Rocca Fellowship which partially supported J.F. Hiller in his doctoral studies. We also thank Phill-Seung Lee of the Finite Element Research Group, Department of Mechanical Engineering, M.I.T., for his help in preparing this paper.

\section{Appendix A. Application of the proposed error measure to a Timoshenko cantilever beam}

In this appendix, we illustrate the error measure of Section 2.4 when applied to a Timoshenko cantilever beam [3].

\section{A.1. Structure considered}

We consider a straight cantilever beam occupying the domain $\Omega=[0 ; L]$. The thickness of the beam is denoted 


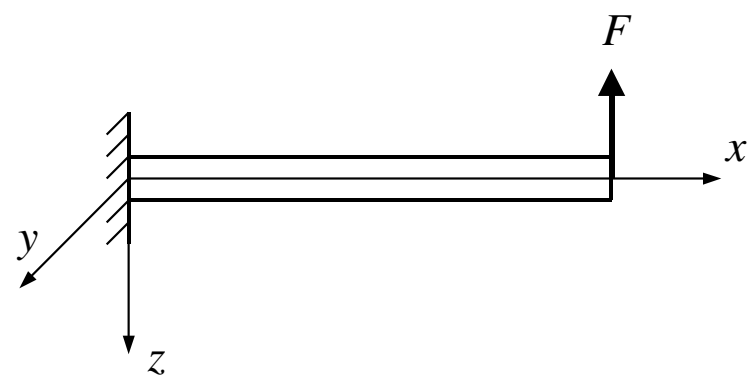

Fig. 12. Beam problem definition.

by $t$ and its width is 1 . The beam is clamped at $x=0$. The loading consists of a concentrated force $F$ applied at the free end, as shown in Fig. 12. The beam is made of a linear isotropic elastic material with Young's modulus $E$ and shear modulus $G$.

The beam is modelled using the Timoshenko beam theory. The governing equations for the beam are:

$$
\left\{\begin{array}{l}
Q=G t\left(\frac{\mathrm{d} w}{\mathrm{~d} x}+\Phi\right) \\
M=E I \frac{\mathrm{d} \Phi}{\mathrm{d} x}
\end{array}\right.
$$

where $Q$ is the shear force, $M$ is the bending moment, $w$ and $\Phi$ are the $z$ direction translation and $y$ axis rotation and $I=t^{3} / 12$. In this beam structure only two strains are present:

$\left\{\begin{array}{l}\epsilon_{x x}=z \frac{\mathrm{d} \Phi}{\mathrm{d} x} \\ \gamma_{x z}=\Phi+\frac{\mathrm{d} w}{\mathrm{~d} x}\end{array}\right.$

The exact solution is

$$
\left\{\begin{array}{l}
w=\frac{F}{E I}\left[\frac{(x-L)^{3}}{6}-\frac{L^{2} x}{2}+\frac{L^{3}}{6}\right]-\frac{F}{G t} x \\
\Phi=-\frac{F}{E I}\left[\frac{(x-L)^{2}}{2}-\frac{L^{2}}{2}\right]
\end{array}\right.
$$

In particular, the tip displacement and rotation are

$$
\left\{\begin{array}{l}
w(L)=-\frac{F L^{3}}{3 E I}-\frac{F L}{G t} \\
\Phi(L)=\frac{F L^{2}}{2 E I}
\end{array}\right.
$$

and the exact strains of the mathematical model are given by

$$
\left\{\begin{array}{l}
\gamma_{x z}=-\frac{F}{G t} \\
\epsilon_{x x}=\frac{F z}{E I}(L-x)
\end{array}\right.
$$

\section{A.2. Finite element solution with $N$ elements}

We model the beam structure with $N$ equally sized mixed-interpolated finite elements, with linear variations in the transverse displacement $w$ and section rotation $\Phi$ and constant element transverse shear strain (i.e. tied at the element mid-point, see [2]). It can be seen that in the finite element solution the shear strain is predicted exactly at all points and the bending strain is predicted exactly at the mid-point of each element. We can make use of this property combined with the fact that the strains in the element with nodes $n+1$ and $n$ verify [2]

$\left\{\begin{array}{l}\gamma_{x z}^{\mathrm{AS}}=\frac{w_{n+1}-w_{n}}{L / N}+\frac{\Phi_{n+1}+\Phi_{n}}{2} \\ \epsilon_{x x}=\frac{N z}{L}\left(\Phi_{n+1}-\Phi_{n}\right)\end{array}\right.$

to obtain expressions for the nodal displacements and rotations. With some algebra, we find that the nodal displacements and rotations are given by

$$
\left\{\begin{array}{l}
w_{n}=\frac{F L^{3}}{E I N^{3}}\left(\frac{n^{3}}{6}-\frac{N n^{2}}{2}+\frac{n}{12}\right)-\frac{F L n}{G t N} \\
\Phi_{n}=\left(-\frac{1}{2} n^{2}+N n\right) \frac{F L^{2}}{E I N^{2}}
\end{array}\right.
$$

\section{A.3. Proposed error measure}

Using the definition of our error measure in Eq. (12), with some algebra we obtain that $\operatorname{EM}\left(U, U_{h}\right)=F^{2} L^{3} /$ $2 E t^{3} N^{2}$, all of it coming from the bending energy since the transverse shear is predicted exactly at all points.

From Eq. (A.4), we see that the strain energy of the exact solution is

$E(U, U)=F^{2}\left(\frac{2 L^{3}}{E t^{3}}+\frac{L}{2 G t}\right)$

Hence the relative error for this problem is

$$
\begin{aligned}
\operatorname{RE}\left(U, U_{h}\right) & =\frac{\operatorname{EM}\left(U, U_{h}\right)}{E(U, U)}=\frac{\frac{F^{2} L^{3}}{2 E t^{3} N^{2}}}{F^{2}\left(\frac{2 L^{3}}{E t^{3}}+\frac{L}{2 G t}\right)} \\
& =\frac{1}{N^{2}} \frac{1}{4+\frac{E}{G}\left(\frac{t}{L}\right)^{2}}
\end{aligned}
$$

Therefore we have

$\operatorname{RE}\left(U, U_{h}\right) \leqslant \frac{1}{4 N^{2}}=\frac{h^{2}}{4 L^{2}}$

where $h=L / N$ is the element size. The fact that we could find an upper bound for the relative error of the form $C h^{2}$ with $C$ independent of $t$ and the optimal order 
of convergence, which is 2 for the linear element considered, indicates that there is no locking. ${ }^{4}$

\section{A.4. Alternative error measures}

We have mentioned that our proposed error measure of Eq. (12) is a norm for the strain error but is in general not a norm for the displacements. This might be a difficulty for specific problems where we need to evaluate the error in the displacements with great accuracy. In this section, we comment on three alternative error measures that are norms for the displacements.

\section{A.4.1. Displacement-based strain energy}

In this section, we consider the strain energy as an alternative error measure:

$\overline{\operatorname{EM}}\left(U, U_{h}\right)=E\left(U-U_{h}, U-U_{h}\right)$

Considering our problem, we find after some algebra, using Eq. (A.7), that the contribution to $\overline{\mathrm{EM}}$ from bending is

$\overline{\mathrm{EM}}_{\mathrm{b}}=\frac{F^{2} L^{3}}{2 E t^{3} N^{2}}$

and the contribution from shear is

$\overline{\mathrm{EM}}_{\mathrm{s}}=\frac{\left(4 N^{2}-1\right)}{N^{4}} \frac{G F^{2} L^{5}}{2 E^{2} t^{5}}$

It should be noted that the contribution from bending is the same for this alternative error measure as for our proposed error measure, of course, because the bending strains are not mixed-interpolated in the element considered. The shear contribution differs however: in our proposed error measure there is no contribution from shear. It can be argued that from the point of view of the engineer, our error measure is in this regard more logical: considering that the shear strains are predicted exactly at all points in the structure, it is unexpected to have a non-zero contribution to the error measure coming from shear.

The relative error is

$\overline{\operatorname{RE}}\left(U, U_{h}\right)=\frac{1}{N^{2}} \frac{1+\frac{4 N^{2}-1}{N^{2}} \frac{L^{2}}{t^{2}} \frac{G}{E}}{4+\frac{E}{G} \frac{t^{2}}{L^{2}}}$

This expression cannot be put in the form

for $t$ small enough, $\quad \overline{\operatorname{RE}}\left(U, U_{h}\right) \leqslant C \frac{1}{N^{2}}$

\footnotetext{
${ }^{4}$ Of course, in this problem only shear locking could be present.
}

with $C$ a constant independent of the thickness $t$. As a matter of fact, for $t$ small enough, $\left(1+\left(\left(4 N^{2}-1\right) / N^{2}\right) \times\right.$ $\left.\left(L^{2} / t^{2}\right)(G / E)\right) /\left(4+(E / G)\left(t^{2} / L^{2}\right)\right)$ behaves as $\left(\left(4 N^{2}-\right.\right.$ 1) $\left./ 4 N^{2}\right)\left(L^{2} / t^{2}\right)(G / E)$ and goes to infinity as $t$ goes to zero. Note that it is therefore the contribution to the error measure coming from shear that prevents Eq. (A.15) from holding. Hence, using the error measure $\overline{\mathrm{EM}}$ we would obtain the impression that the element shear locks, although in reality the shear strain is predicted exactly at all points. ${ }^{5}$

\section{A.4.2. $A_{m}+A_{b}$}

Another error measure that is used for bendingdominated problems is [8]

$\operatorname{EM}^{*}\left(U, U_{h}\right)=A_{m}\left(U-U_{h}, U-U_{h}\right)+A_{\mathrm{b}}\left(U-U_{h}, U-U_{h}\right)$

One advantage of this error measure is that it defines a norm equivalent to the norm for the space $\mathscr{V}$, as proven in [8]. Using Eqs. (A.12) and (A.13), we obtain immediately that

$\operatorname{EM}^{*}\left(U, U_{h}\right)=\frac{F^{2} L^{6}}{t^{6} N^{2}}\left[\frac{1}{2 E}+\frac{G}{2 E^{2}} \frac{4 N^{2}-1}{N^{2}}\right]$

and therefore the relative error in this norm is

$$
\begin{aligned}
\operatorname{RE}^{*}\left(U, U_{h}\right) & =\frac{\operatorname{EM}^{*}\left(U, U_{h}\right)}{A_{m}(U, U)+A_{\mathrm{b}}(U, U)} \\
& =\frac{1}{N^{2}} \frac{\frac{1}{2 E}+\frac{G}{2 E^{2}} \frac{4 N^{2}-1}{N^{2}}}{2+\frac{t^{4}}{2 L^{4} G}}
\end{aligned}
$$

We can put this expression in the form

$\operatorname{RE}^{*}\left(U, U_{h}\right) \leqslant C \frac{1}{N^{2}}$

with $C$ a constant independent of the thickness $t$. Hence in this error measure we find, similarly to our proposed error measure, that there is no locking.

One disadvantage of using $\operatorname{EM}^{*}\left(U, U_{h}\right)$ as an error measure is that this measure only applies in the case of bending-dominated problems and we need to resort to a different error measure to assess the performance of a finite element formulation in membrane-dominated or mixed (intermediate) cases. Another disadvantage of this approach is that the physical meaning of $\mathrm{EM}^{*}$ is not immediate.

\footnotetext{
${ }^{5}$ We implemented the error measure $\overline{\mathrm{EM}}$ of Eq. (A.11) for shell problems and observed in this case also that the relative error $\overline{\mathrm{RE}}$ can take surprisingly large values even when a reasonably accurate solution has been reached.
} 


\section{A.5. $E M+\|\cdot\|_{L^{2}}^{2}$}

We mentioned in Section 2.4 that our proposed norm could be modified into a norm for the displacements by adding to it the $L^{2}$ norm of the displacements, in the form

$$
\begin{aligned}
\operatorname{EM}^{\prime}\left(U, U_{h}\right)= & \operatorname{EM}\left(U, U_{h}\right)+C \int_{0}^{L}\left(w-w_{h}\right)^{2} \mathrm{~d} x \\
& +C^{\prime} \int_{0}^{L}\left(\Phi-\Phi_{h}\right)^{2} \mathrm{~d} x
\end{aligned}
$$

where we denote by $w$ the translational degrees of freedom and $\Phi$ the rotational degrees of freedom.

From Eqs. (A.3) and (A.7), we evaluate

$$
\int_{0}^{L}\left(w-w_{h}\right)^{2} \mathrm{~d} x=\frac{F^{2} L^{7}}{E^{2} I^{2}} \frac{394 N^{3}-8 N}{N^{7}}
$$

and

$$
\int_{0}^{L}\left(\Phi-\Phi_{h}\right)^{2} \mathrm{~d} x=\frac{F^{2} L^{5}}{120 E^{2} I^{2}} \frac{1}{N^{4}}
$$

As expected, both terms vary with $N$ as $1 / N^{4}$, whereas the error in the strains varies as $1 / N^{2}$. Hence we have

$$
\begin{aligned}
\operatorname{EM}^{\prime}\left(U, U_{h}\right)= & \frac{F^{2} L^{3}}{2 E t^{3} N^{2}}+C \frac{F^{2} L^{7}}{E^{2} I^{2}} \frac{394 N^{3}-8 N}{N^{7}} \\
& +C^{\prime} \frac{F^{2} L^{5}}{120 E^{2} I^{2}} \frac{1}{N^{4}}
\end{aligned}
$$

We can also evaluate the $L^{2}$ norms for the translations and rotations of the exact solution

$$
\int_{0}^{L} w^{2} \mathrm{~d} x=\frac{F^{2} L^{3}}{420 E^{2} I^{2} G^{2} t^{2}}\left(11 L^{4} G^{2} t^{2}+77 L^{2} E I G t+140 E^{2} I^{2}\right)
$$

and

$$
\int_{0}^{L} \Phi^{2} \mathrm{~d} x=\frac{2}{15} \frac{F^{2} L^{5}}{E^{2} I^{2}}
$$

which show that these quantities vary like $t^{-6}$ as $t$ goes to zero. We could propose to use $C=t^{3} E / L^{4}$ and $C^{\prime}=$ $t^{3} E / L^{2}$. Defining

$$
\operatorname{RE}^{\prime}\left(U, U_{h}\right)=\frac{\operatorname{EM}\left(U, U_{h}\right)+C\left\|w-w_{h}\right\|_{L^{2}}^{2}+C^{\prime}\left\|\Phi-\Phi_{h}\right\|_{L^{2}}^{2}}{E(U, U)+C\|w\|_{L^{2}}^{2}+C^{\prime}\|\Phi\|_{L^{2}}^{2}}
$$

this choice of constants would result in

$$
\begin{aligned}
\operatorname{RE}^{\prime}\left(U, U_{h}\right)=( & \frac{L^{3}}{2 t^{3} N^{2}}+\frac{L^{3} t^{3}}{I^{2}} \frac{394 N^{3}-8 N}{N^{7}} \\
& \left.+\frac{t^{3}}{L^{2}} \frac{L^{5}}{120 I^{2}} \frac{1}{N^{4}}\right) /\left(\left(\frac{2 L^{3}}{t^{3}}+\frac{L E}{2 G t}\right)\right. \\
& +\frac{t^{4}}{420 L I^{2} G^{2}}\left(\frac{11 L^{4} G^{2}}{t}+77 \frac{L^{2} E I G}{t^{2}}\right. \\
& \left.\left.+140 \frac{E^{2} I^{2}}{t^{3}}\right)+t^{3} \frac{2}{15} \frac{L^{3}}{I^{2}}\right)
\end{aligned}
$$

All three terms of the numerator and denominator vary like $1 / t^{3}$ and the numerator varies with $N$ as $1 / N^{2}$, which implies that we have, for $t$ small enough,

$\operatorname{RE}^{\prime}\left(U, U_{h}\right) \leqslant C \frac{1}{N^{2}}$

The disadvantage of the error measure $\mathrm{EM}^{\prime}$ is that it does not have a clear physical meaning and for some complex situations the constants $C$ and $C^{\prime}$ may not be easy to select.

\section{References}

[1] Brezzi F, Fortin M. Mixed and hybrid finite element methods. Springer Verlag; 1991.

[2] Bathe KJ. Finite element procedures. Prentice-Hall; 1996.

[3] Bathe KJ. The inf-sup condition and its numerical evaluation for mixed finite element methods. Comput Struct 2001;79:243-52, 971.

[4] Chapelle D, Bathe KJ. Fundamental considerations for the finite element analysis of shell structures. Comput Struct 1998;66(1):19-36, 711-2.

[5] Lovadina C. Energy estimates for linear elastic shells. In: Bathe KJ, editor. Computational fluid and solid mechanics. Elsevier Science; 2001. p. 330-1.

[6] Auricchio F, Beirão da Veiga L, Lovadina C. Remarks on the asymptotic behaviors of Koiter shells. Comput Struct 2002;80:735-45.

[7] Lee PS, Bathe KJ. On the asymptotic behavior of shell structures and the evaluation in finite element solutions. Comput Struct 2002;80:235-55.

[8] Chapelle D, Bathe KJ. The finite element analysis of shells-fundamentals. Springer Verlag; 2003.

[9] Bathe KJ, Chapelle D, Lee PS. A shell problem 'highlysensitive' to thickness changes. Int $\mathbf{J}$ Numer Meth Eng, in press.

[10] Iosilevich A, Bathe KJ, Brezzi F. On evaluating the inf-sup condition for plate bending elements. Int J Numer Meth Eng 1997;40:3639-63.

[11] Bathe KJ, Iosilevich A, Chapelle D. An inf-sup test for shell finite elements. Comput Struct 2000;75:439-56.

[12] Chapelle D, Bathe KJ. The inf-sup test. Comput Struct 1993;47:537-45.

[13] El-Abbasi N, Bathe KJ. Stability and patch test performance of contact discretizations and a new solution algorithm. Comput Struct 2001;79:1473-86. 
[14] Dvorkin EN, Bathe KJ. A continuum mechanics based four-node shell element for general nonlinear analysis. Eng Comput 1984;1:77-88.

[15] Bucalem M, Bathe KJ. Higher-order MITC general shell elements. Int J Numer Meth Eng 1993;36:3729-54.

[16] Bathe KJ, Dvorkin EN. A formulation of general shell elements - the use of mixed interpolation of tensorial components. Int J Numer Meth Eng 1986;22:697-722.

[17] Hiller JF, Bathe KJ. On higher-order-accuracy points in isoparametric finite element analysis and an application to error assessment. Comput Struct 2001;79:1275-85.

[18] Verfürth R. A review of a posteriori error estimation and adaptive mesh-refinement techniques. Wiley-Teubner; 1996.

[19] Babuška I, Strouboulis T. The finite element method and its reliability. Oxford University Press; 2001.
[20] Bathe KJ, Iosilevich A, Chapelle D. An evaluation of the MITC shell elements. Comput Struct 2000;75:1-30.

[21] Reissner E. The effect of transverse shear deformations on the bending of elastic plates. J Appl Mech 1945;67:A69-77.

[22] Mindlin RD. Influence of rotary inertia and shear on flexural motion of isotropic elastic plates. J Appl Mech 1951;18:31-8.

[23] Pitkäranta J, Matache AM, Schwab C. Fourier mode analysis of layers in shallow shell deformations. Comput Meth Appl Mech Eng 2001;190:2943-75.

[24] Chapelle D, Bathe KJ. The mathematical shell model underlying general shell elements. Int J Numer Meth Eng 2000;48:289-313.

[25] Bathe KJ, Lee PS, Hiller JF. Towards improving the MITC9 shell element. Comput Struct 2003;81:1085-97. 